\title{
A NOTE ON A BROKEN-CYCLE THEOREM FOR HYPERGRAPHS
}

\author{
MARTIN TRINKS \\ Hochschule Mittweida, University of Applied Sciences \\ Faculty Mathematics / Sciences / Computer Science \\ Technikumplatz 17, 09648 Mittweida, Germany \\ e-mail: trinks@hs-mittweida.de
}

\begin{abstract}
Whitney's Broken-cycle Theorem states the chromatic polynomial of a graph as a sum over special edge subsets. We give a definition of cycles in hypergraphs that preserves the statement of the theorem there.

Keywords: Broken-cycle Theorem, hypergraphs, cycles, chromatic polynomial, graph polynomials.
\end{abstract}

2010 Mathematics Subject Classification: 05C31, 05C15.

\section{REFERENCES}

[1] C. Berge, Hypergraphs, Vol. 45 (North-Holland Mathematical Library, NorthHolland, 1989).

[2] K. Dohmen, A broken-circuits-theorem for hypergraphs, Arch. Math. 64 (1995) 159162. doi:10.1007/BF01196637

[3] F.M. Dong, K.M. Koh, and K.L. Teo, Chromatic polynomials and chromaticity of graphs (World Scientific Publishing, 2005).

[4] P. Jégou and S.N. Ndiaye, On the notion of cycles in hypergraphs, Discrete Math. 309 (2009) 6535-6543. doi:10.1016/j.disc.2009.06.035

[5] M. Trinks, Graph polynomials and their representations, PhD Thesis, Technische Universität Bergakademie Freiberg, (2012).

[6] H. Whitney, The coloring of graphs, Proc. Natl. Acad. Sci. USA 17(2) (1931) 122125. doi:10.1073/pnas.17.2.122

[7] H. Whitney, A logical expansion in mathematics, Bull. Amer. Math. Soc. 38(8) (1932) $572-579$. doi:10.1090/S0002-9904-1932-05460-X 